\title{
Investigation of the Prognosis of 28 Patients with Retained Placenta After Delivery
}

\author{
Hirokazu Naoi ${ }^{1}$, Keiichi Kumasawa ${ }^{1,}$, Hitomi Nakamura ${ }^{1}$, Aiko Nishikawa ${ }^{1}$, Tadashi Kimura ${ }^{1}$, \\ Kazuhide Ogita ${ }^{2}$ \\ ${ }^{1}$ Department of Obstetrics and Gynecology, Osaka University, Suita City, Japan \\ ${ }^{2}$ Department of Obstetrics and Gynecology, Rinku General Medical Center, Izumisano City, Japan
}

Email address:

kumasawa@gyne.med.osaka-u.ac.jp (K. Kumasawa),naoihirokazu@yahoo.co.jp (H. Naoi), hitomi@gyne.med.osaka-u.ac.jp (H. Nakamura), aikoriver@yahoo.co.jp (A. Nishikawa), tadashi@gyne.med.osaka-u.ac.jp (T. Kimura), k-ogita@rgmc.izumisano.osaka.jp (K. Ogita)

${ }^{*}$ Corresponding author

\section{To cite this article:}

Hirokazu Naoi, Keiichi Kumasawa, Hitomi Nakamura, Aiko Nishikawa, Tadashi Kimura, Kazuhide Ogita. Investigation of the Prognosis of 28 Patients with Retained Placenta After Delivery. Journal of Gynecology and Obstetrics. Vol. 4, No. 2, 2016, pp. 7-11.

doi: $10.11648 /$ j.jgo.20160402.11

Received: March 2, 2016; Accepted: March 28, 2016; Published: April 13, 2016

\begin{abstract}
A retained placenta after delivery can lead to bleeding and infection or other serious complications. There are 2 approaches for managing this condition: one is observation until spontaneous expulsion or resorption of the placenta, the other is active intervention such as manual removal, dilation and evacuation, uterine artery embolization, and hysterectomy. In this study, we investigated cases of retained placenta in our institution. We reviewed cases of retained placenta treated in our institution to investigate the associated risk factors and provide indications for its clinical management. 28 patients with retained placenta were treated in our institution from April 1, 2008 to March 31, 2012. Clinical background, management, and prognosis were retrospectively reviewed. Induced or augmented labor, primipara, pregnancy after reproductive technology and post-term delivery after 41 weeks were found to be risk factors for retained placenta in this study. Patients with severe complications showed a significantly larger maximum diameter of the retained placenta than those without serious complications. Patients showing partial retention of the placenta with a maximum diameter $<3 \mathrm{~cm}$ are at a reduced risk of serious complications and should be managed by observation.
\end{abstract}

Keywords: Retained Placenta, Postpartum Hemorrhage, Intrauterine Infection

\section{Introduction}

Obstetric hemorrhage still remains a leading cause of maternal death, and retained placenta is one of the strong contributor. Retained placenta is the failure of placental deliver. It complicates $2-3 \%$ of vaginal deliveries, and was reported to be associated with postpartum hemorrhage and intrauterine infection leading to infection. The management of retained placenta is complex, in particular in cases of partial retention. Thus far the standard treatment of retained placenta is manual removal. However it is sometimes accompanied with placenta accrete, increta, percreta, leading to massive bleeding and other interventions, such as uterine artery embolism or hysterectomy. Therefore if observation seems to be useful, careful observation is sometimes selected. especially in the case of partially retained placenta. There is currently no standard procedure for the management of partially retained placenta and the treatment strategy is left to the discretion of the physician. They have to take into consideration risk-benefit balance between waiting and active intervention. Therefore, in the present study, we analyzed 28 cases of wholly or partially retained placenta treated in our institution to provide guidelines for the management of this condition. Several factors such as previous history of retained placenta, preterm birth, history of intrauterine manipulation, induced or augmented labor, and preeclampsia have been reported as risk factors associated with retained placenta [1-6]. Recently still birth has been added to the group of risk factors [6]. One of the aim of this study was to investigate patients' backgrounds were investigated, and the risk factors associated 
with the incidence of retained placenta in our institution were analyzed in the study. In addition to the previously reported risk factors, we focused on the gestational week of delivery, and analyzed the relation between retained placenta and gestational week. The other aim of our study was to suggest a criteria of the residual placental size within which observation was available. We analyzed the relation between the largest length of residual placenta and following complications, such as infection and massive bleeding.

\section{Materials and Methods}

All of 4243 deliveries in our institution from April 1, 2008 to March 31, 2012 were enrolled in this study. Among them the total number of deliveries in our institution was 4243, and 28 patients (28 cases) were diagnosed as retained placenta after birth. Patient's background, risk factors, prognosis, and clinical management were investigated for the 28 patients. Retained placenta was diagnosed after 30 minutes after delivery of infant [1]. This is a reasonable limit when the third stage of labor is actively managed. In all cases, oxytosin and fundal pressure for uterine contraction were performed, and controlled cord traction was sometimes drawn gently. All patients received ultrasonographic examination The maximum diameter of the residual placenta was measured by ultrasonography. The largest width of retained placenta was measured as the maximum diameter. Figure 1 shows an example of retained placenta measured by ultrasonography.

We performed statistical analysis on clinical factors of the 28 cases and extracted the factors associated with serious complications (massive bleeding and intrauterine infection). Risk factors for retained placenta were identified by International Classification of Disease, $10^{\text {th }}$ revision (ICD-10). We analyzed gestational age, delivery history (primiparous or multiparous), history of spontaneous abortion, history of artificial abortion, pregnancy assisted by reproductive technology, induced or augmented labor, history of cesarean section, history of retained placenta and the maximum diameter of the retained portion. Massive bleeding was defined as blood loss of more than $1000 \mathrm{~mL}$ within 24 hours after birth, and intrauterine infection was defined as maternal fever with temperatures above $38^{\circ} \mathrm{C}$ caused by uterine endometritis. Statistical analysis was performed using Statistical Package for Social Sciences (SPSS Inc, Chicago, IL). For analyzing maximum diameter of retained placenta, in relation to complications, 28 patients were divided into two groups. Group A included 14 cases involving severe complications (massive bleeding or intrauterine infection), and group B included 14 cases without complications. Comparison among groups was performed with the chi-squared test and $P<.05$ was considered to indicate a statistically significant result.

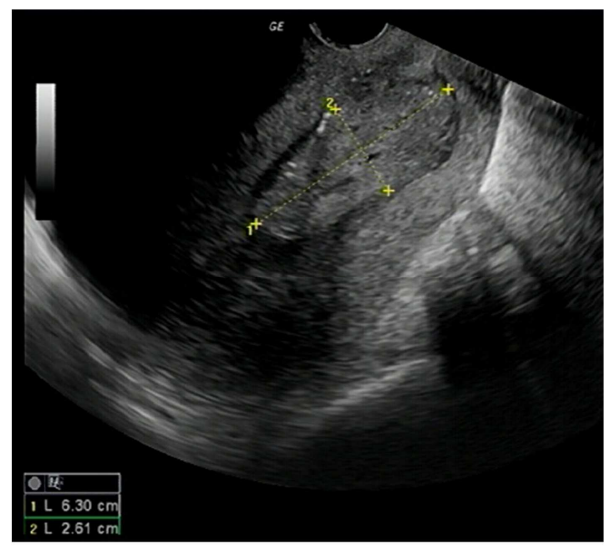

Fig. 1. Example of retained placenta measured observed using ultrasonography.

\section{Results}

There were 28 cases (the case group) of retained placenta during the study period and the total number of deliveries in our institution without them (the control group) was 4215. Of 28 patients of retained placenta, 20 had placenta retention after live birth (three cases were after cesarean section), four had retention after artificial abortion, and four were after intrauterine fetal death or spontaneous abortion. Figure 2 shows the details of the 28 cases of retained placenta treated in our institution.

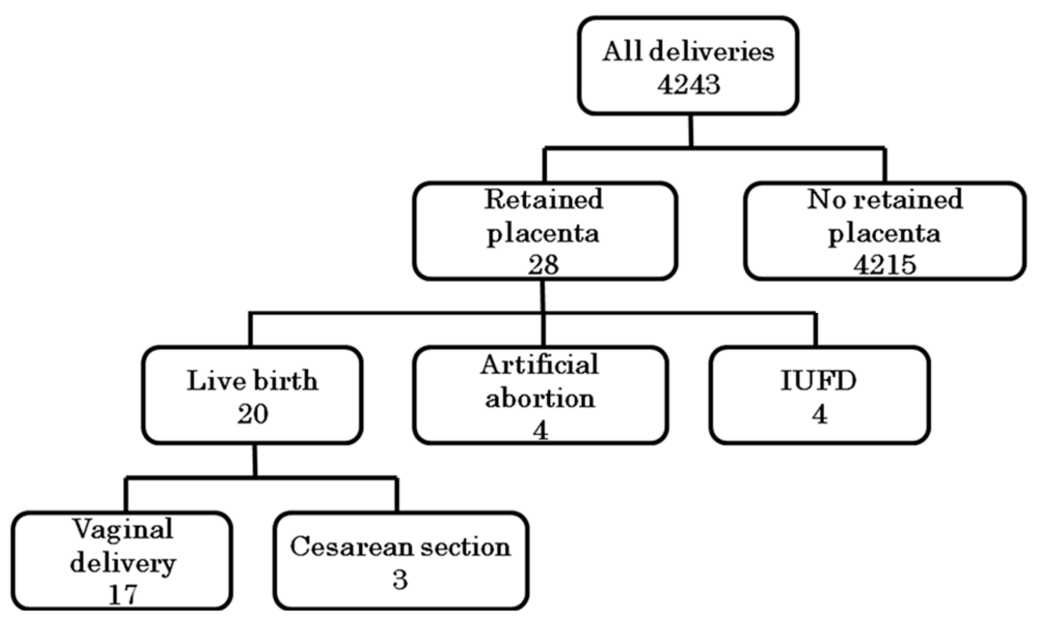

Fig. 2. Details of the 28 cases of retained placenta treated in our institution. 
In nine cases (32.1\%), spontaneous expulsion or resorption of the partially retained placenta occurred during observation in the outpatient clinic. Active intervention was required in nineteen patients $(67.9 \%)$. Of these nineteen patients, five $(17.9 \%)$ underwent manual removal; five (17.9\%), simple hysterectomy; eight (28.6\%), dilation and evacuation; and one $(3.6 \%)$, uterine artery embolization. Eleven patients (38.3\%) required active intervention after massive bleeding, and four patients $(14.3 \%)$ were treated after intrauterine infection (including cases showing both symptoms). Table 1 shows the clinical background of the 28 patients included in the study.

Table 1. Clinical Background and Perinatal Prognosis of the 28 Patients.

\begin{tabular}{|c|c|c|c|}
\hline characteristic & Case $(n=28)$ & Control $(n=4215)$ & P value \\
\hline Primiparous & $20(71.4 \%)$ & $1635(38.8 \%)$ & $<.005$ \\
\hline $\begin{array}{l}\text { History of spontaneous } \\
\text { abortion }\end{array}$ & $6(21.4 \%)$ & $813(19.3 \%)$ & .78 \\
\hline $\begin{array}{l}\text { History of artificial } \\
\text { abortion }\end{array}$ & $6(21.4 \%)$ & $754(17.9 \%)$ & .63 \\
\hline $\begin{array}{l}\text { *Pregnancy after } \\
\text { reproductive } \\
\text { technology }\end{array}$ & $7(25.0 \%)$ & $143(3.4 \%)$ & $<.005$ \\
\hline $\begin{array}{l}\text { Induced or augmented } \\
\text { labor }\end{array}$ & $15(53.6 \%)$ & $540(12.8 \%)$ & $<.005$ \\
\hline $\begin{array}{l}\text { History of cesarean } \\
\text { section }\end{array}$ & $3(10.7 \%)$ & $527(12.5 \%)$ & .77 \\
\hline $\begin{array}{l}\text { History of retained } \\
\text { placenta }\end{array}$ & $1(3.6 \%)$ & $8(0.2 \%)$ & $<.005$ \\
\hline $\begin{array}{l}\text { Over-term delivery } \\
\text { after } 41 \text { gestational } \\
\text { weeks }\end{array}$ & $8(28.6 \%)$ & $364(8.6 \%)$ & $<.005$ \\
\hline Intrauterine fetal death & $4(16.7 \%)$ & $57(1.35 \%)$ & $<0.05$ \\
\hline
\end{tabular}

*Pregnancy after reproductive technology refers to pregnancy by ovulation induction, artificial insemination with husband's semen (AIH), in vitro fertilization-embryo transfer (IVF-ET), and intracytoplasmic sperm injection (ICSI).

Of the 28 patients, twenty (71.4\%) were primiparous, and the proportion was significantly higher than that of the control group $(\mathrm{P}<.005)$. Six $(21.4 \%)$ patients had histories of spontaneous abortion and six (21.4\%) had histories of artificial abortion. Histories of dilation and evacuation were difficult to assess because they were self-reported, but the rate of retained placenta group was similar to that of the control group and there was no significant difference between the two groups (spontaneous abortion: $\mathrm{P}=.78$, artificial abortion: $\mathrm{P}=.63)$. Seven patients $(25.0 \%)$ got concieved by infertility treatment (e.g. ovulation induction, artificial insemination with husband's semen, in vitro fertilization and embryo transfer, and intracytoplasmic sperm injection), and there was significant difference compared with the control group $(\mathrm{P}<.005)$. Fifteen patients $(53.6 \%)$ were affected by retained placenta after induced or augmented labor, and the ratio was also significantly higher than the control group $(\mathrm{P}<.005)$. Four patients $(16.7 \%)$ were affected by IUFD, which was significantly higher than control groups. Only one of the 28 patients $(3.6 \%)$ had a history of retained placenta, and the proportion was calculated to be significantly higher.
But the number of the case was so small that we would like to leave this matter for future research. Preeclamptic women were not involved in the 28 women with retained placenta.

Next, the 28 patients were divided into 2 groups as shown above: group A consisted of 14 cases with severe complications, and group B included 14 cases without complications. Figure 3 shows the distribution of the maximum diameters of the retained placenta in each group.

maximum diameter $(\mathrm{mm})$

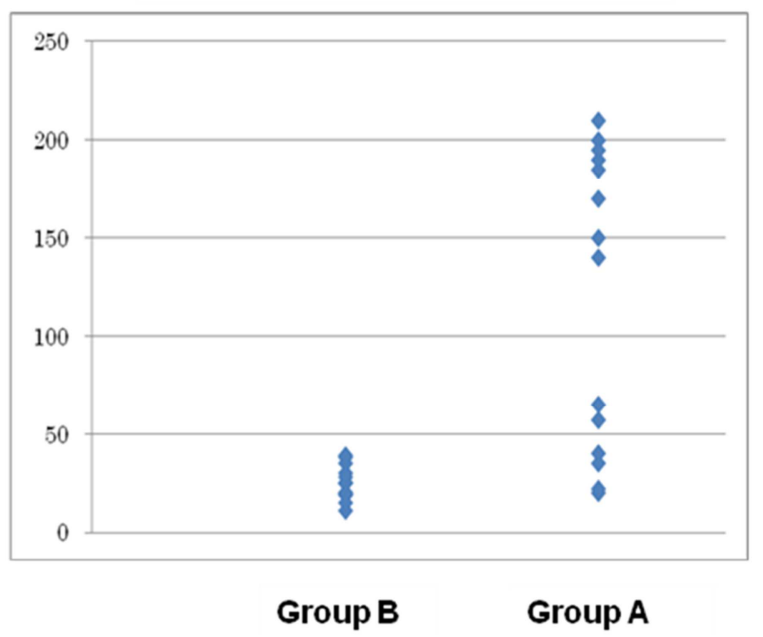

Fig. 3. Distribution of the maximum diameter of retained placenta of each group.

The maximum diameter of the retained placenta in group $\mathrm{A}$ varied from 20 to $195 \mathrm{~mm}$ (average, $120 \mathrm{~mm}$; standard deviation, $75.0 \mathrm{~mm}$ ), and that of group B varied from 11 to $39 \mathrm{~mm}$ (average, $24.9 \mathrm{~mm}$; standard deviation, $8.4 \mathrm{~mm}$ ). The maximum diameter in group A was found to be significantly larger than that of group B $(P<.001)$. These results indicate that a larger maximum diameter was associated with an increased risk of complications.

\section{Discussion}

The risk factors for retained placenta reported ever include history of retained placenta, premature delivery, history of dilation and evacuation, induced or augmented labor, and preeclampsia. Endler M and colleagues reported that the risk of retained placenta is increased in subsequent pregnancies if there is a history of retained placenta (OR: 12.6) [7]. However, among the 28 cases in our institution, only one patient had a history of retained placenta and the risk could not be discussed on the basis of our data. Endler $\mathrm{M}$ and colleagues also reported that premature delivery and preeclampsia are also risk factors (ORs 2.35 and 1.37, respectively) [5]. However, our population included only one case of preterm delivery (between 24 to 36 gestational weeks), and there were no case of preeclampsia. Therefore, the risk associated with preterm delivery and preeclampsia could not be estimated. Moreover, about 40 years ago Green LK and colleagues reported that uterine malformation or is 
associated with retained placenta [8], which was not confirmed in our study population. Ergometrine for preventing atonic bleeding in the $3^{\text {rd }}$ stage of labor was also reported to be related with retained placenta [9]. However in our study erogometrine was not used for patients with retained placenta. Therefore we can't discuss the risk factor. In every delivery in our institution oxytosin was used after delivery of infants. The proportion of induced or augmented labor in the present 28 cases of retained placenta (fifteen cases, $53.6 \%$ ) was significantly higher than that of the control group $(\mathrm{P}<.005)$. These results indicate that among reported risk factors only induced or augmented labor was a risk factor associated with retained placenta in our study. Additionally, as mentioned above, primipara and pregnancy after reproductive technology were found to be risk factors in our institution. Moreover, among 28 patients, eight $(28.6 \%)$ delivered their infants after 41 gestational weeks. The ratio was significantly higher than that of the control group $(\mathrm{P}<.005)$. Therefore, delivery after 41 gestational weeks was also suggested to be a risk factor for retained placenta in our study. Obstetricians need to pay more attention in the $3^{\text {rd }}$ stage of labor of women with 41 gestational weeks. In our study ratio of IUFD in retained placenta group (16.7\%) was obviously higher. Endler $\mathrm{M}$ and colleagues reported that still birth increased the occurrence of retained placenta (OR: 1.71) [5].

As mentioned above, comparison of the maximum diameter between group A with massive bleeding or intrauterine infection and group B without these adverse events showed that the maximum diameter of patients in group A was significantly larger than that of patients in group B. Additonally group A was composed of two subgroups as shown in Figure 3. One group (group A-1) included patients with almost wholly retained placenta whose maximum diameter was greater than $10 \mathrm{~cm}$, whereas the other group (group A-2) was considered to include patients with partially retained placenta whose maximum diameter was approximately $5 \mathrm{~cm}$. On the other hand, all patients in group $\mathrm{B}$ were thought to be affected by partially retained placenta because the maximum diameters were all smaller than $4 \mathrm{~cm}$. Therefore, we compared group A-2 to group B because both groups were composed of patients with partially retained placenta. Fig 4 shows the maximum diameter of the retained placenta in both groups.

The maximum diameter of the retained placenta in group A-2 ranged from 20 to $65 \mathrm{~mm}$ (average, $40 \mathrm{~mm}$; standard deviation, $18.0 \mathrm{~mm}$ ), and that of group B from 11 to $39 \mathrm{~mm}$ (average, $24.9 \mathrm{~mm}$; standard deviation, $8.4 \mathrm{~mm}$ ). The maximum diameter in group A-2 was found to be significantly larger than that of group $\mathrm{B}(P<.05)$. These results indicate that a larger maximum diameter was also associated with an increased risk of complications in the partially retained placenta group.

Next, we determined the critical point for medical intervention based on the size of the retained placenta. A cutoff value was set, and Figure 5 shows the receiver operating characteristic (ROC) curve indicating the sensitivity and specificity of each value.
As shown in Figure 5, a cutoff value of $3.25 \mathrm{~cm}$ was considered adequate. When the cutoff value was set at $3 \mathrm{~cm}$, the sensitivity was $80.0 \%$, specificity was $84.6 \%$, positive predictive value was $85.7 \%$, and negative predictive value was $78.6 \%$. These values were shown to be comparable thus far. These results suggest that if the placenta is retained partially and the maximum diameter is less than $3 \mathrm{~cm}$, the risk of severe complications such as massive hemorrhage and intrauterine infection is low, and patients should be treated by observation. This study was performed retrospectively and the number of applied cases was not large because the incidence of retained placenta is not high. In fact, in our institution, only $0.66 \%$ (28 cases in 4243 deliveries) of pregnant women were complicated with retained placenta. Therefore, we are planning the prospective cohort study including a larger number of patients from many hospitals based on this study.

\section{maximum diameter $(\mathrm{mm})$}

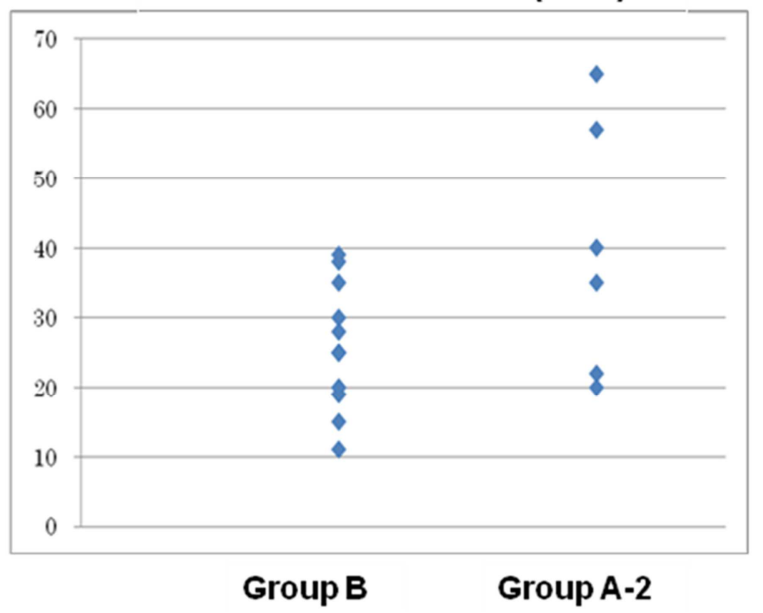

Fig. 4. Distribution of the maximum diameter of retained placenta in groups $A-2$ and $B$.

RoC Curve

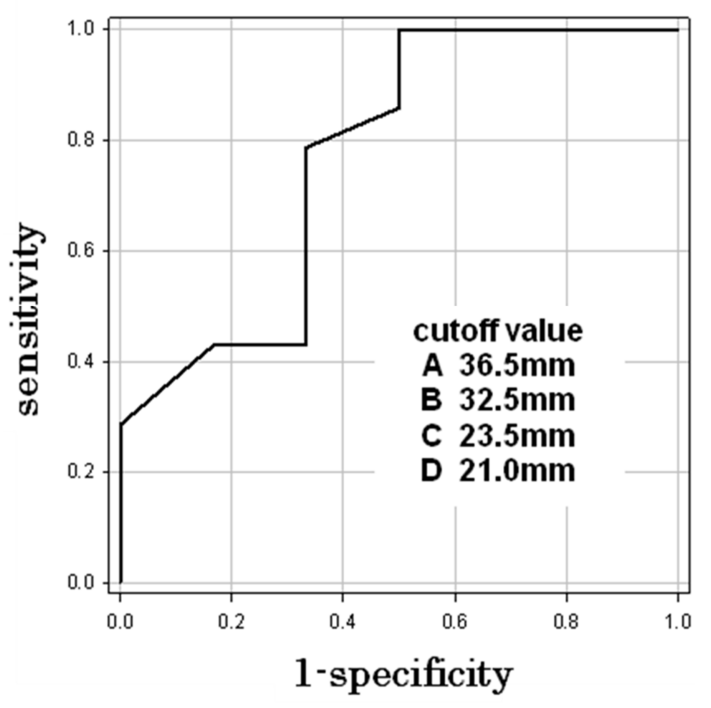

Fig. 5. ROC curve corresponding to each cutoff value. 


\section{Conclusions}

Risk factors of retained placenta were primiparous, infertility treatment, induced or augmented labor, history of retained placenta, and IUFD as previously reported. Additionally in this study delivery after 41 gestational weeks was risk factor. Patients with partial retention of the placenta with a maximum diameter $<3 \mathrm{~cm}$ are at a reduced risk of serious complications and careful observation can be adopted.

\section{References}

[1] Deneux-Tharaux C, Macfarlane A, Winter C, et al "Policies for manual removal of placenta at vaginal delivery: variations in timing within Europe." BJOG, Vol 116, pp 119-124, Jan 2009

[2] Akol AD, Weeks AD. "Retained Placenta: will medical treatment ever be possible?" Acta Obstet Gynecol Scand. Jan 2016 Epub ahead of print

[3] Coviello EM, Grantz KL, Huang CC, et al. "Risk factors for retained placenta." Am J Obstet Gynecol. Vol 213 pp: 864. e1-864.e11. Dec. 2015

[4] Ashwal E ${ }^{1}$, Melamed N, Hiersch L, et al. "The incidence and risk factors for retained placenta after vaginal delivery - a single center experience."

[5] Endler M, Saltvedt S, Cnattingius S, et al. "Retained placenta is associated with pre-eclampsia, stillbirth, giving birth to a small-for-gestational-age infant, and spontaneous preterm birth: a national register-based study." BJOG. vol 121 pp1462-1470, Nov, 2014

[6] Panpaprai P, Boriboonhirunsarn D, et al. "Risk factors of retained placenta in Siriraj Hospital," J Med Assoc Thai. Vol90, pp. 1293-1297, July 2007

[7] Endler M, Grünewald C, Saltvedt S. "Epidemiology of retained placenta: oxytocin as an independent risk factor," Obstet Gynecol, Vol 119, pp.801-809, April 2012

[8] Green LK, Harris RE. "Uterine anomalies. Frequency of diagnosis and associated obstetric complications," Obstet Gynecol, Vol 47, pp. 427-429, April 1976

[9] C. M. Choy, W. C. Lau, W. H. Tam, P. M. Yuen. “A randomized controlled trial of intramuscular syntometrine and intravenous oxytocin in the management of the third stage of labour" BJOG, Vol 109, pp. 173-177, 2002

[10] Orji E, Agwu F, Loto O, Olaleye O. "A randomized comparative study of prophylactic oxytocin versus ergometrine in the third stage of labor."Int J Gynaecol Obstet, Vol 101, pp 129-132, May 2008 